\title{
TENSIONES Y DISTENSIONES EN TORNO A LA CIUDADANÍA Y FORMACIÓN CIUDADANA: COMPARACIÓN DE LOS SIGNIFICADOS DE PROFESORES Y ESTUDIANTES SECUNDARIOS EN LA REGIÓN DE VALPARAÍSO
}

\author{
ANDREA FLANAGAN BÓRQUEZ* \\ GUSTAVO CERDA GONZÁLEZ ${ }^{* *}$ \\ DANIELA LAGOS GONZÁLEZ \\ SANDRA RIQUELME TORRIJOS ${ }^{* * * *}$
}

RESUMEN

El presente estudio tiene por objetivo comprender los significados que poseen profesores y estudiantes de establecimientos secundarios de la región de Valparaíso, respecto a la ciudadanía y la formación ciudadana. Se entiende formación ciudadana como el proceso previo al ejercicio de la ciudadanía, y por tanto, de la participación (electoral y discursiva) que los sujetos realizan en una sociedad determinada. Para alcanzar los objetivos, se entrevistó a estudiantes y profesores de las distintas dependencias (municipalizadas, particular subvencionado y particular pagado) de las provincias de Los Andes, San Felipe, San Antonio, Petorca y Valparaíso. Los datos fueron analizados bajo el método cualitativo de la Teoría Fundamentada (Grounded Theory), pues a través del análisis de contenido categorial se buscaba conocer y reconocer el desarrollo de la ciudadanía al interior de los liceos/colegios y la influencia de los actores en este proceso. Los principales resultados del estudio muestran diferencias en la forma de concebir la ciudadanía y la formación ciudadana de los estudiantes y los profesores, así como en las formas que poseen los diversos establecimientos secundarios para abordar y educar en el tema.

PALABRAS CLAVE: EDUCACIÓN CIUDADANA, ENSEÑANZA MEDIA CHILENA, CIUDADANÍA

* Escuela de Psicología, Universidad de Valparaíso, Valparaíso, Chile. Correo electrónico: andrea.flanagan@uv.cl.

** Psicólogo, Universidad de Valparaíso.

Correo electrónico: gustavo.cerda77@yahoo.es.

*** Psicóloga educacional, Universidad de Valparaíso.

Correo electrónico: dani.lagos@gmail.com.

**** Psicóloga, Universidad de Valparaíso.

Correo electrónico: sandra.crt@gmail.com.

Este estudio se efectuó gracias al apoyo de la Dirección de Investigación de la Universidad de Valparaíso, Chile (Proyecto de Investigación DIPUV 24/06). Los autores agradecen la colaboración de Sonia Astudillo y Ana Luisa Muñoz, quienes contribuyeron en el desarrollo de la presente investigación. 


\title{
TENSÕES E DISTENSÕES SOBRE CIDADANIA E FORMAÇÃO CIDADÃ: COMPARAÇÃO DOS SIGNIFICADOS DE PROFESSORES E ESTUDANTES SECUNDÁRIOS NA REGIÃO DE VALPARAÍSO
}

\section{Resumo}

Este estudo teve como objetivo compreender os significados de cidadania e formação cidadã de professores e estudantes de estabelecimentos secundários da região de Valparaíso. Entende-se por Formação Cidadã o processo anterior ao exercício da cidadania, portanto, a participação (eleitoral e discursiva) que os sujeitos realizam em uma determinada sociedade. Para atingir esses objetivos, foram entrevistados estudantes e professores das diferentes dependências (municipalizadas, particulares subvencionadas e particulares pagas) das províncias de Los Andes. San Felipe, San Antonio, Petorca e Valparaíso. Os dados foram analisados pelo método qualitativo da Teoria Fundamentada (Grounded Theory). Através da análise de conteúdo por categoria, buscou-se conhecer e reconhecer o desenvolvimento da cidadania no interior dos liceus/colégios e a influência dos atores neste processo. Os principais resultados do estudo mostram diferenças na forma de conceber a cidadania e a formação cidadã por estudantes e professores, bem como nas formas que os diversos estabelecimentos secundários abordam e promovem a educação nesta temática.

PALAVRAS CHAVE: EDUCAÇÃO CIDADÃ, ENSINO MÉDIO CHILENO, CIDADANIA

\section{STRESSES AND STRAINS ON THE CITIZENSHIP AND CIVIC EDUCATION: COMPARING THE MEANINGS OF TEACHERS AND HIGH SCHOOL STUDENTS IN REGION OF VALPARAÍSO}

\begin{abstract}
The present study aimed to understand the meanings that have teachers and students of high school in the region of Valparaíso, about citizenship and civic education. Civic Education is defined as the process prior to the exercise of citizenship and thus participation (electoral and discursive) that subjects performed in a given society. To achieve the objectives, interviews were conducted with students and teachers from various departments (municipal, private subsidized and private) in the provinces of Los Andes, San Felipe, San Antonio, Petorca and Valparaiso. The data were analyzed by the qualitative method of Grounded Theory, because through the categorical content analysis was aimed to know and recognize the development of citizenship within the high schools/colleges and the influence of the actors in this process. The main results of the study show differences in the way of thinking about citizenship and civic education students and teachers, as well as forms that have various secondary institutions to approach and educate on the subject.
\end{abstract}

KEY WORDS: CITIZEN EDUCATION, SECONDARY EDUCATION, CITIZENSHIP 


\section{CONTEXTO DE LA CIUDADANÍA Y LA FORMACIÓN CIUDADANA EN EL ESCENARIO EDUCATIVO CHILENO}

LA ACTUAL SOCIEDAD CHILENA ha debido superar una serie de conflictos y tensiones internas debido a su historia de violaciones a los derechos humanos, civiles y a las condiciones democráticas, transgresiones que se dieron durante el gobierno militar y que transformaron las significaciones de ciudadanía hasta entonces desarrolladas (Moulian, 1997; Portales, 2000; Cerda et al., 2004).

Con el término de dicho gobierno se generaron una serie de expectativas sobre el nuevo rol que ocuparía la sociedad civil, no obstante los gobiernos concertacionistas optaron por una estrategia de priorizar la gobernabilidad del país por sobre las reivindicaciones sociales e históricas (Portales, 2000; Cerda et al., 2004).

En este contexto, el ejercicio de la ciudadanía se vio dificultado con las tensiones de convivir en un constante escenario político que cuestionaba el verdadero estado de derecho (Portales, 2000), evitando los componentes esenciales del ejercicio de la ciudadanía como son el diálogo, la discusión y el debate, perdiéndose la perspectiva sobre el ejercicio democrático, como un espacio de encuentro de visiones de mundo, de país y de comunidad (Cerda et al., 2004).

A lo anterior, se suma el hecho de que dentro de esta democracia de acuerdos, las negociaciones se realizaban entre expertos o técnicos políticos, con lo cual se dificultó aún más el ejercicio ciudadano de la sociedad civil (Portales, 2000), y alejó a los partidos políticos de la cotidianeidad social (Cerda et al., 2004). Esta lógica esencialmente cupular, consolida en la sociedad civil una percepción de que su participación ciudadana poco puede influir en la construcción y operación de un proyecto país (Moulian, 1997; Cerda et al., 2004). Todo esto fue influyendo en las prácticas ciudadanas, constatándose un progresivo desinterés de parte de los jóvenes en participar de los circuitos formales de la política (Cerda et al., 2004).

Respecto a la formación ciudadana en la educación chilena, algunos antecedentes muestran que éste es un proceso que aún se encuentra en desarrollo. Es así que en un estudio internacional realizado por la IEA, y citado por la Comisión del MINEDUC (2004a), se define el nivel de los conocimientos cívicos en los jóvenes chilenos a los 14 y a los 17-18 años, así como la comparación de los mismos en el contexto internacional. 
Los resultados de esta medición indican que los jóvenes chilenos poseen un bajo nivel de conocimiento sobre las temáticas relacionadas con ciudadanía y política (MINEDUC, 2004a; Cerda et al., 2004).

Ahora bien, la formación ciudadana además de ser una temática transversal del actual currículo educativo, y específica para la materia de historia y ciencias sociales, se fortalece especialmente, en otras tres asignaturas, a saber: lenguaje y comunicación, orientación/consejo de curso y filosofía (Decretos Supremos de Educación N40, 1996 y №220, 1998; respectivamente, MINEDUC, 2004a).

En lo que respecta a las opciones metodológicas para impartir formación ciudadana, entre éstas se incluye «una asignatura propia», «integrada a ciencias sociales», «integrada a todas las asignaturas» (enfoque transversal), o impartida «extracurricularmente». En Chile se ha consolidado un modelo mixto de implementación desde la asignatura de historia y ciencias sociales e integrada transversalmente en todas las materias (MINEDUC, 2004a).

La problemática abordada en el presente estudio se enmarca en la tesis que plantea que las sociedades, y especialmente en Chile, se vivencia una crisis de la validación de los sistemas democráticos, que queda demostrado por la disminución de la participación ciudadana en los espacios formales de representación en Chile (Thezà, 2003). Con más de veinte años de educación en democracia, los jóvenes se han distanciado del ejercicio de voto en las distintas instancias de elecciones locales, regionales y nacionales (MINEDUC, 2004a). Ni la mayor libertad de expresión en los centros educativos ni los contenidos curriculares incorporados a través de la educación cívica —-después de haber sido eliminados por el gobierno militar - han logrado aumentar la participación (Garretón, 2000). Del mismo modo, la Sexta Encuesta Nacional de Juventud (INJUV, 2010) señala que el 23,8\% de los jóvenes entre 15 y 29 años está insatisfecho con la democracia, porcentaje que se incrementa con la edad, educación y localidades urbanas; por otra parte, el $24,1 \%$ de los jóvenes dentro del mismo rango etario se encuentra satisfecho con el sistema

Por otro lado, diversas investigaciones sobre conocimientos cívicos en jóvenes chilenos han demostrado, que en comparación a otros países, los jóvenes chilenos poseen en promedio menor cantidad de conocimientos sobre estas materias, diferenciándose de los promedios internacionales, indicando con ello una mayor debilidad formativa precisamente en temáticas excluidas del programa educativo por el gobierno militar (MINEDUC, 2004a; ICCS, 2010). 
Estos elementos explican, entonces, el interés de los últimos gobierno chilenos en este tema, que se refleja, entre otras iniciativas, en la creación de la Comisión Especial del Ministerio de Educación de Chile sobre la Formación Ciudadana, a través de la cual se confluyen una serie de reflexiones sobre la participación de los jóvenes en política, el proceso de formación ciudadana en centros educativos y el rol que los actores de la comunidad educativa estarían cumpliendo y deberían cumplir dentro de este marco (MINEDUC, 2004a). Esto no es menor, pues los centros educativos juegan un importante papel en este proceso, ya que los jóvenes pasan una gran cantidad de horas en estos establecimientos, configurándose como el espacio responsable de entregar, mediar y evaluar en el desarrollo del aula los contenidos del programa curricular (Andrade, 2001; en Astudillo y Cerda, 2006), que tienen relación con la formación ciudadana como uno de los Objetivos Fundamentales Transversales que se incorporaron a través de la Reforma Educacional durante la década pasada (MINEDUC, 2004b).

Como plantea Martínez (1998), los docentes, actores claves del proceso de formación en educación, forman parte de la influencia que los jóvenes reciben en el desarrollo de su aprender a ser, esto último entendido según lo planteado por Delors (1996), como la construcción de sus identidades —quiénes están siendo y qué sienten—, promoviendo en ellos condiciones personales que les permitan ser ciudadanos activos dentro de su entorno y capaces del comprender al otro, reconociendo la diversidad así como la solidaridad y la conciencia de la existencia de diferencias, a favor de fortalecer la democracia en la sociedad.

En lo contextual, la región de Valparaíso se distingue por ser la tercera con mayor población de jóvenes, con un 10\% del total nacional (INJUV, 2006) y al mismo tiempo es la tercera suscripción electoral más grande del país (Servicio Electoral, 2006). Por esta razón los procesos de formación ciudadana en esta región marcan niveles de alcance importante en la población juvenil.

Basados en los antecedentes antes mencionados, surgen una serie de cuestionamientos que dan forma a este estudio, a saber: ¿cuáles son los significados que dan los estudiantes y profesores a la ciudadanía y a la formación ciudadana en los establecimientos educacionales secundarios de la región de Valparaíso?, ¿qué tipo de formación ciudadana se imparte en los diversos establecimientos educacionales secundarios?, ¿cuál es el acercamiento pedagógico al tema?, ¿cuáles son las tensiones existentes entre los discursos de los distintos actores entrevistados? 
La relevancia del tema está dada por la prioridad que los últimos gobiernos han dado a la calidad de la ciudadanía en nuestra sociedad civil, ya que de esta depende la constitución de una democracia plena (MINEDUC, 2004a). Es en este marco donde se hace necesario profundizar en los significados que poseen estudiantes y profesores sobre el tema, dando cuenta del peso relativo que posee la educación formal en los nuevos ciudadanos, explicitando a la vez las dinámicas particulares de este proceso de formación en el desarrollo cívico social. Junto a ello, es importante señalar que la temática ha sido escasamente estudiada en la región de Valparaíso, no existiendo hasta el momento modelos que describan y profundicen en la comprensión del fenómeno, por lo que el presente estudio constituye una innovación en el área.

\section{DEBATES Y REVISIÓN DE CONCEPTOS EN TORNO A LA CIUDADANÍA}

Para comprender las distintas visiones existentes acerca de la ciudadanía, se presentan a continuación diversas concepciones, cada una de las cuales determina la manera en que se lleva a cabo la promoción de la ciudadanía, así como las prácticas ciudadanas. La primera visión se basa en el análisis desarrollado por Cerda et al. (2004) desde el cual se define ciudadanía en base al debate liberal-comunitarista; mientras que el segundo modelo postulado por Dueñas (2001) aborda la ciudadanía desde diversas dimensiones, con derechos y deberes ciudadanos característicos. A partir del análisis de ambos modelos, se expone la definición de ciudadanía que será considerada en este estudio.

\section{a) Debate liberal-comunitarista}

Cerda et al. (2004) plantean que la noción de ciudadanía ha cambiado en relación a cómo se concibe desde dos paradigmas: el liberalismo y el comunitarismo.

Ciudadanía desde el liberalismo: Desde este enfoque, la ciudadanía se refiere esencialmente a la entrega de derechos cuya expresión política se ve reflejada en el ejercicio del voto. De esta manera la ciudadanía correspondería al status que se adquiere gracias a la posesión de derechos, por lo tanto, son los derechos los que otorgan ciudadanía. Desde esta perspectiva los derechos individuales se superponen sobre cualquier noción de bien común; por lo tanto, el bien de la sociedad no justifica la violación de estos derechos. La formación para la ciudadanía desde el liberalismo entonces, se basa en lograr que el individuo 
adquiera la autonomía y la capacidad para decidir moralmente, aprenda de las instituciones y de las leyes existentes.

Ciudadanía desde el comunitarismo: Según este paradigma, las personas están determinadas por sus vínculos sociales; por lo tanto, para entender la conducta humana hay que acudir a sus contextos socioculturales e históricos. En este sentido, la ciudadanía se refiere esencialmente a la participación política, puesto que es allí donde el individuo se compromete con la sociedad. Es así que la formación en ciudadanía se basaría en un bagaje de actitudes, valores e ideas que habiliten a las personas para participar en los asuntos públicos, reforzando así los lazos hacia el interior de la comunidad.

Ciudadanía desde un enfoque integrador: Desde este enfoque, la noción de ciudadanía debe incorporar tanto el desarrollo de las capacidades que favorezcan la autonomía del individuo, como también la creación de condiciones que propicien la formación de lazos en la sociedad. En este sentido, lo que da sentido y legitimidad a la posesión de derechos individuales sería la pertenencia a una comunidad.

\section{b) Ciudadanía desde una mirada sistémica}

Dueñas (2001) propone un modelo en el cual la ciudadanía está formada por diversas dimensiones, cada una de las cuales dando origen a diferentes modos de ejercer la ciudadanía. Estas dimensiones son:

Ciudadanía política: Define la relación que establecen los individuos con el poder; por lo tanto involucra los derechos civiles y políticos.

Ciudadanía social: Se refiere a la inserción del individuo en la sociedad en igualdad y equidad de oportunidades. Contiene los derechos económicos y sociales.

Ciudadanía individual: Se basa en la libertad del individuo para decidir autónomamente e incluye los derechos individuales o libertades personales.

Ciudadanía emancipatoria: Se refiere a la legítima aspiración de transformar las estructuras sociales, las formas de vida y la organización económica con el fin de construir un mundo más libre y justo para todos. Involucra, por tanto, los derechos de cambio de la sociedad.

Ciudadanía cultural: Reconoce a las personas, grupos o pueblos como portadoras de identidades étnicas, éticas y estéticas particulares que superan la tensión entre identidad y diversidad. Se realiza en los derechos propios de cada cultura. 
Ciudadanía comunicacional: Involucra el reconocimiento del otro como interlocutor legítimo y con identidad propia, abierto al conocimiento y a la información circundante en esta sociedad globalizada. Si se analizan estas dimensiones a la luz de los paradigmas descritos en el apartado anterior, es posible distinguir los diferentes paradigmas que están a la base de ellas, y que, por tanto, han prevalecido en distintas épocas históricas en nuestro país.

A partir de lo anterior, el concepto de ciudadanía será entendido como una condición ligada siempre a un contexto histórico particular, que determina una serie de valores, actitudes y principios éticos que guían la manera en que el individuo actúa y se desenvuelve en un espacio social. La pertenencia a una comunidad legitima una serie de derechos y deberes que permiten la coexistencia en un mundo diverso, los cuales varían de acuerdo a las transformaciones propias de la evolución de nuestra sociedad.

\section{FORMACIÓN CIUDADANA}

Existen diversas maneras de concebir la formación ciudadana; cada una de estas formas va a determinar la manera en que la institución educativa articule sus prácticas con el fin de formar ciudadanamente a sus educandos (Cerda et al., 2004). Estos autores señalan cuatro formas de entender la formación ciudadana, que se describen a continuación:

Formación para la ciudadanía política: Se sustenta en la concepción liberalista acerca de la ciudadanía y está vinculada a la educación cívica tradicional. Está centrada en formar estudiantes capaces de desarrollar su autonomía moral y que manejen conocimientos acerca de las instituciones democráticas y los derechos individuales (igualdad legal y política, derecho a voto).

Formación para la ciudadanía social: Corresponde a una visión más amplia, ya que además de incluir los derechos civiles y políticos, incorpora los derechos económicos, sociales, culturales y medioambientales como esenciales para desarrollar la vida democrática. Desde esta perspectiva se abordan temas contingentes como la pobreza, el cual pasa a ser un imperativo moral para alcanzar la igualdad como ciudadanos.

Formación para la ciudadanía desde una postura crítica: Incorpora el tema de la distribución del poder haciendo énfasis en la capacidad para cuestionar, problematizar y transformar las prácticas educativas y el currículum con el fin de promover el cambio social. De esta 
manera cuestiona el carácter reproductivo que presenta la formación ciudadana en las prácticas docentes cuyo propósito sería que los estudiantes se adapten a la sociedad. Frente a esto propone estimular a los intelectos para que desafíen a las fuerzas sociales políticas y económicas que dominan el mundo. Desarrollar este «coraje cívico» tiene sus bases en la dimensión emancipatoria de la ciudadanía.

Formación para la ciudadanía activa: Este enfoque da énfasis a la participación de los ciudadanos en el proceso de «autoconstrucción» de la sociedad. Esto significa que no basta con otorgar derechos, sino que además es necesario promover la participación ciudadana para ejercer poder en las decisiones que afectan el bien común. Esta participación constituye un acto donde se asumen responsabilidades individuales y colectivas en pos de lograr un proyecto social común, fundado en una imagen socialmente construida de un «Nosotros». Esta noción está fuertemente ligada al paradigma comunitarista, puesto que considera que es en el espacio público donde el individuo se constituye como ciudadano. Para formar ciudadanamente bajo esta perspectiva, se requiere que las escuelas se transformen a nivel organizativo a modo de construir una institución donde exista un sentido de pertenencia a la comunidad escolar por parte de todos los actores, fomentando los vínculos sociales, la responsabilidad con el otro y la participación, donde todos se identifiquen con una historia y un proyecto común. Es importante mencionar que desde esta última mirada se entenderá la formación ciudadana para el presente estudio.

\section{CIUDADANÍA, PODER Y CURRÍCULO OCULTO}

Los enfoques anteriores no dejan de ser orientaciones con intereses ideológicos posibles de analizar y develar, como plantea Torres (1998). Uno de los mitos más importantes al interior de los sistemas educativos neoliberales es la supuesta neutralidad de los mismos sistemas y los enfoques teóricos que los sustentan. Supuesto que cumple, según algunos autores radicales, la función de reproducir el estado de las actuales condiciones de vida en beneficio de algunos y contra del bienestar de muchos/as (Giroux, 1983).

Esto nos advierte la importancia de identificar precisamente las lógicas ideológicas que operan a la base de las políticas educativas y las prácticas escolares, o sea, la forma en como los actores sociales construyen significativamente su realidad, sus expectativas, valoraciones y sueños; lo que Torres (1998) llamará ideologías. 
Por ende, las propuestas teóricas descritas en el apartado anterior, no escapan a estas construcciones, y a estos intereses ideológicos, pudiéndose reconocer algunas propuestas más explícitas que otras, y donde Cerda et al. (2004), por ejemplo, realizan un esfuerzo importante por presentar un meta-análisis de los enfoques, pero donde al final no pueden escapar de presentar una propuesta propia, que busca rescatar elementos de diversas posiciones ideológicas, connotadas positivas por ellos/as.

Así, cada una de las propuestas tendría consecuencias en las relaciones sociales y de poder que se constituyen en cada comunidad educativa, constituyendo un sentido común que traspasa los años escolares y que trasmite una forma de estar en el mundo (Torres, 1998; Giroux, 1992), una forma de entender la ciudadanía y las prácticas relacionadas con ella.

Esto no es menor, ya que estos sentidos comunes no se imponen homogéneamente en los microespacios (familias o instituciones escolares), sino que conviven entre sí, se contaminan y se transforman (Torres, 1998), constituyéndose en el discurso y la práctica como fisuras posibles de profundizar a favor de la transformación social. Algunos autores (Berumen y Levinson, 2007) han confirmado esta diversidad de significados existentes desde los discursos de programas mexicanos de formación ciudadana, nacionales e internacionales y que influyen las prácticas escolares, observando dos grandes polos: i) desde el fomento de las libertades individuales/privacidad; ii) hasta el fomento del pensamiento crítico/actitud protagónica. Ambos polos se condicen con el esquema liberal versus comunitario presentado anteriormente.

El presente artículo nos permite precisamente ver reflejados algunos de los significados que transitan en diversas comunidades educativas sobre el concepto de ciudadanía y la formación ciudadana, lo que entendemos como una expresión del Currículum Oculto (Torres, 1998) y nos permite vislumbrar la forma en que se proyecta la Sociedad Civil desde el aparato escolar.

Nuevamente Berumen y Levinson (2007) aportan con tres grandes categorías de significados posibles:

i) Valores perdidos: un relato que considera que se vive actualmente una desintegración social general, con un aumento de la violencia, corrupción, divorcios y pérdida de autoridad de los adultos. Para su solución aluden a recuperar valores como el respeto, la honestidad 
y la obediencia, por medio de una formación religiosa o catequizadora. Reclaman que el sentido jerárquico se encuentra en tensión y la perdida de respeto de parte de los jóvenes.

ii) Ciudadano crítico: apunta a fomentar hábitos democráticos profundos hacia una cultura política. Para esto se llama a una participación más activa, frente a un clientelismo social para el Estado; un uso del diálogo respetuoso pero crítico e interrogante; fomentar el proceso de debate racional para llegar a opciones independientes; y valora la equidad sobre la jerarquía.

iii) Rendición de cuentas: influido por agentes internacionales, es un relato que valora la transparencia administrativa pública y formas nuevas de evaluación; la definición de metas de transparencia y calidad son transversales; y la promoción de la construcción de una nueva subjetividad sobre la necesidad de rendir cuentas.

$\mathrm{Al}$ menos, las dos primeras categorías son posibles de reconocer en los enfoques ya descritos, y llama la atención en la diversidad de significados con que transitan concepciones claves como participación, democracia y/o ciudadanos/as (Berumen y Levinson, 2007).

\section{MÉTODO}

El estudio se enmarcó dentro del interaccionismo simbólico, pues se buscaba comprender el fenómeno respecto a los significados que poseen estudiantes y profesores de establecimientos de enseñanza secundaria de la región de Valparaíso, respecto a las nociones de ciudadanía y de formación ciudadana.

A partir de la experiencia vivida por los propios actores, en sus propios contextos, se pudo conocer lo que ellos consideran real y lo que dirige e influye sus acciones, sus pensamientos y sus sentimientos (McMillan y Schumacher, 2005:401).

El diseño de investigación es de tipo emergente, pues es necesario que éste se adapte al fenómeno de estudio, pudiendo ser modificado durante el desarrollo de la investigación, a fin de lograr un conocimiento más profundo del fenómeno de estudio (Salamanca y MartínCrespo, 2007). 
Los establecimientos participantes fueron los siguientes.

TABLA 1: ESTABLECIMIENTOS Y ACTORES PARTICIPANTES DEL ESTUDIO

\begin{tabular}{|c|c|c|c|}
\hline Provincia & Establecimiento & Tipo de dependencia & Participantes \\
\hline \multirow{2}{*}{ Los Andes } & $\begin{array}{c}\text { Colegio San Sebastián } \\
\text { de Los Andes }\end{array}$ & Particular Subvencionado & $\begin{array}{c}\text { Estudiantes } \\
\text { Profesores }\end{array}$ \\
\hline & $\begin{array}{c}\text { Liceo Técnico } \\
\text { Amancay }\end{array}$ & Municipalizado & Estudiantes \\
\hline \multirow{2}{*}{ San Felipe } & $\begin{array}{c}\text { Colegio Alonso } \\
\text { de Ercilla }\end{array}$ & Particular Subvencionado & Estudiantes \\
\hline & $\begin{array}{l}\text { Liceo Politécnico } \\
\text { Roberto Humeres } \\
\end{array}$ & Municipal & Estudiantes \\
\hline San Antonio & $\begin{array}{c}\text { Colegio Santo Domingo } \\
\text { Country School }\end{array}$ & Particular Pagado & Estudiantes \\
\hline Petorca & Colegio Andrés Bello & Particular Subvencionado & Profesores \\
\hline \multirow[t]{2}{*}{ Valparaíso } & $\begin{array}{c}\text { Liceo Eduardo } \\
\text { de la Barra }\end{array}$ & Municipal & Profesores \\
\hline & Liceo René Descartes & Particular Subvencionado & Profesores \\
\hline
\end{tabular}

Se construyeron pautas de entrevistas a fin de conocer y comprender los significados de estudiantes y profesores, las cuales se orientaron a indagar sobre los siguientes temas: concepciones y conceptualizaciones sobre ciudadanía y formación ciudadana en el contexto actual; influencia del sistema de la educación secundaria en la ciudadanía y formación ciudadana; y tipo de ciudadanía y formación ciudadana que se promueve y expresa en el establecimiento entrevistado.

En el caso de los estudiantes, se organizaron Grupos de discusión conformados por entre 8 y 12 alumnos/as de $I^{\circ}$ a IV ${ }^{\circ}$ año de enseñanza secundaria y de características heterogéneas en cuanto a rendimiento académico, género, edad, participación y antigüedad en el establecimiento.

Respecto de los profesores, se organizaron cuatro Grupos Focales (uno por cada establecimiento), procurando que en su conformación estuvieran presentes principalmente docentes de los subsectores reconocidos por el Ministerio de Educación chileno como los de mayor influencia en la formación ciudadana, a saber: lenguaje y comunicación; filosofía y psicología; historia y ciencias sociales; y comprensión del medio natural, social y cultural. ${ }^{1}$

1 Este último ramo se divide en la educación secundaria entre: física, química y biología; frente a lo cual el estudio sólo consideró el subsector de biología, por su vinculación curricular con los contenidos de ecología y las relaciones de la especie humana como grupo y su ambiente. 
La utilización de esta técnica se basó en la utilidad de las exploraciones iniciales, en donde es apropiada y pertinente para obtener opiniones y captar los significados de los actores, a partir de lo que surge de la discusión (Mella, 2000).

Los datos fueron analizados en base a la Teoría Fundamentada («Grounded Theory») propuesta por Strauss y Corbin (2002). A través de este método, se codificó cada una de las entrevistas efectuadas a los participantes, para posteriormente agruparlas en categorías, conceptos o constructos a fin de establecer las diferencias y semejanzas con respecto a una u otra categoría que el investigador identifique.

\section{Principales Resultados}

A continuación se presentan los principales resultados obtenidos en el presente estudio respecto a la ciudadanía y luego sobre la formación ciudadana.

\section{a) Ciudadanía}

Fue posible apreciar que los participantes tienen apreciaciones distintas respecto a la definición y entendimiento de ésta. Por un lado, los profesores consideran que un ciudadano se define como tal si está informado de los acontecimientos que ocurren en la sociedad y si participa activamente de su entorno por medio de votaciones, ya sea a nivel nacional o, en el caso de los alumnos, a través de la elección del centro de estudiantes. Por otro lado, los estudiantes participantes definen a un ciudadano en términos de los contenidos que debe poseer, por ejemplo contar con valores como el cuidado de la ciudad, conocer y obedecer normas y leyes y saber procedimientos característicos de un país como lo es el sufragio. Por otra parte se destaca que estos contenidos forman parte de los objetivos que tienen instituciones como escuelas y colegios para formar a un ciudadano. En general, se señala que estos contenidos durante los años de enseñanza deben ser asimilados por los estudiantes, con el objetivo de formar personas que a futuro respondan de manera satisfactoria al medio en el que están insertos y participen de manera activa en él. De acuerdo a las definiciones de ciudadano que entregan los estudiantes se presenta la siguiente cita: «En el colegio por lo menos a mí me pasan educación ciudadana y nos enseña por ejemplo cuando uno tiene que ir a votar o por ejemplo derecho a voto y todas esas cosas... Pero aparte también es como for- 
mar valóricamente a la persona, cuidando el medio ambiente, que el ciudadano tiene que cuidar todo eso, son temas que incumben a los ciudadanos, delincuencia, los colegios, los asaltos todo eso».

Un segundo tema emergente fue sobre las condiciones para ser ciudadano, es decir, las condiciones específicas para ser considerado como tal. Los docentes y estudiantes coinciden en señalar que el pertenecer a una ciudad, país o localidad, participar activamente de éstas, tener 18 años de edad, estar inscritos en los registros electorales y ejercer el derecho a voto, son criterios que determinan que una persona sea ciudadano.

Otro aspecto que emergió del discurso fueron las valoraciones negativas y positivas que se atribuyen a los diferentes ciudadanos. Tanto los profesores como los estudiantes destacaron características negativas más que positivas. Los docentes señalan que las características negativas del ciudadano en general son el individualismo y los prejuicios, mientras que los alumnos señalan que existe una diferencia entre las personas que viven en el campo y las que habitan la ciudad, manifestando que estas últimas son más peligrosas que las primeras. Por otra parte, se describe a los chilenos como agresivos, discriminadores, pesimistas, racistas, delincuentes, individualistas, irrespetuosos e indiferentes frente a temas relevantes. Respecto a los aspectos positivos del ciudadano en general los alumnos señalan la solidaridad y el cuidado de la ciudad por parte de algunas personas.

Los docentes hacen una distinción hacia los ciudadanos jóvenes, indicando que estos poseen baja participación electoral, actitudes inadecuadas, irrespetuosas y que suelen reclamar por sus derechos sin que cumplan debidamente con sus deberes. Por su parte, los estudiantes definen a este ciudadano como una persona desinteresada de lo que ocurre en su medio o entorno.

Un tercer ciudadano que apareció fue el estudiante, el cual es descrito por los alumnos como antisocial e individualista, sin embargo, se valoran de forma positiva las organizaciones estudiantiles, ya que se reconoce al centro de alumnos como una estructura que sirve de intermediario y representante al interior de las instituciones educacionales.

Un cuarto aspecto visualizado fueron las prácticas de los ciudadanos respecto a las principales formas de participación en su entorno. De acuerdo a esto, se destacan las actividades de voluntariado y ayuda a los demás, los eventos culturales característicos de una localidad y la participación política a través del ejercicio del voto, como las principales formas de expresar la opinión. 
En cuanto al contexto histórico y su influencia en el ciudadano se puede destacar que los participantes del estudio evidencian un cambio en el clima social, en donde la tranquilidad caracterizaba tiempos anteriores, a diferencia de hoy en que el vandalismo identifica la sociedad actual. También se indica que existe una pérdida de control en la familia, en donde se puede visualizar a padres más liberales, flexibles y permisivos, lo que les impide mantener el control de sus hijos. Además se representa a la sociedad actual como más diversa, con mayor tecnología, más individualista y a los jóvenes como capaces de exigir sus derechos, pero también como personas desinteresadas por lo que ocurre en su entorno, irresponsables de sus actos y con vicios dañinos para la salud.

Al momento de hablar sobre los derechos que tiene un ciudadano, docentes y estudiantes coinciden en mencionar el sufragio y la expresión de ideas y opiniones. Sin embargo, los profesores agregan a esta categoría el derecho a participar en su comunidad, la igualdad y equidad de derechos para los distintos habitantes.

Respecto a los deberes de los ciudadanos, los docentes señalan el mantenerse informado, respetar al otro, ser una persona activa dentro de su entorno. Por su parte, los estudiantes indican como deberes el cuidado del medio ambiente, el respeto de normas laborales, el pago de impuestos y la necesidad de superación respecto a su grupo, siendo así un aporte para quienes lo rodean.

Finalmente a los participantes del estudio se les pregunta sobre las características que debe poseer el ciudadano ideal, frente a lo cual señalan la responsabilidad, solidaridad, perseverancia, tolerancia, participativo, informado, crítico y cooperador. Mientras que los estudiantes indican como personas que ellos consideran un ejemplo de ciudadano a sus padres y a Benito Baranda.

\section{b) Formación ciudadana}

De acuerdo al concepto de formación ciudadana, los docentes lo definen como el informar al alumno con el objetivo de que participe activamente de su entorno, aspecto que reconocen falta por desarrollar en los estudiantes. Por otra parte, los alumnos definen formación ciudadana como la enseñanza o instrucción respecto a normas y leyes que rigen el país. De acuerdo al concepto es posible distinguir entre las definiciones de los alumnos ciertos contenidos y objetivos involucrados en la formación ciudadana. Por ejemplo, entre los objetivos se menciona el poder formar a un ciudadano con cierta mentalidad de acuerdo al país 
en que vive, también se indica como objetivo el formar personas que busquen el bien y desarrollo del país y no tan sólo el crecimiento a nivel individual. La siguiente cita es un ejemplo de lo expresado por los estudiantes respecto a los objetivos de la formación ciudadana: «La educación como para hacerlos más partícipes del país en el fondo...También se necesita la formación como en criterio político para poder votar y ser ciudadano...Como formar al ciudadano, como formar la mentalidad que quizás no la tienen, mostrar lo que es ser ciudadano».

En cuanto a los contenidos involucrados específicamente en el espacio escolar tanto estudiantes como docentes expresan que las diferentes instituciones educativas cooperan en la formación de un ciudadano, sin embargo, los alumnos reconocen que esta tarea se podría mejorar.

En cuanto a las valoraciones que docentes y estudiantes atribuyen a los diferentes actores, los docentes describen a los alumnos en sus aspectos negativos como personas desinformadas, que por lo general defienden ciertas causas por un motivo que desconocen, como lo ocurrido con la derogación de la ley LOCE, en donde un gran porcentaje de alumnos participaban de la movilización sin conocer la ley y las consecuencias para la educación. Por otra parte, también se menciona que este estudiante es apático frente a lo que ocurre en la sociedad y a temáticas de contingencia, demostrando su rechazo frente a actividades que involucren estos temas. Además se destaca que los alumnos están acostumbrados a clases de tipo expositivas y al momento de enfrentar actividades de análisis y discusión no son reconocidas por ellos como una instancia de aprendizaje, teniendo como consecuencia una carencia en la capacidad de análisis.

Por otra parte, los alumnos mencionan que los aspectos negativos del estudiante son la irresponsabilidad, indiferencia frente a su aprendizaje, evidenciando desinterés por su educación, teniendo que ser los profesores quienes lo incentiven en temáticas de estudios.

Con respecto a las valoraciones negativas que atribuyen los estudiantes a docentes y directivos se menciona la exclusión de ciertos alumnos, el maltrato verbal y la discriminación, aspectos mencionados principalmente por estudiantes de establecimientos de tipo municipal.

También los participantes del estudio mencionan aspectos positivos de lo que ocurre en el espacio escolar; de acuerdo a esto los estudiantes señalan que en las instituciones educativas existe preocupación por parte de los profesores por enseñar valores a los estudiantes y por su bienestar, existiendo un vínculo entre docentes y alumnos, el cual se caracteriza por la entrega de apoyo. 
Al momento de preguntar a docentes y estudiantes sobre los responsables de la formación ciudadana al interior de las escuelas en general existe acuerdo en mencionar a todos sus actores como responsables, ya sean directivos, docentes, auxiliares, asistentes de la educación y los alumnos, estos últimos principalmente como receptores de las herramientas que los profesores entregan.

En cuanto a las expectativas que docentes y estudiantes tienen de los alumnos existe cierto acuerdo de ambos participantes por destacar la formación de personas con valores como el respeto, la solidaridad, responsables, personas activas y productivas para su comunidad y jóvenes que puedan enfrentar distintas situaciones, lo cual coincide con lo que los participantes del estudio consideran como las funciones de las instituciones educativas en lo que concierne a formación ciudadana.

Respecto a los contenidos involucrados en los currículos de los establecimientos cabe destacar que tanto docentes como estudiantes reconocen la asignatura de historia como la principal materia que abarca temas de formación ciudadana, además de considerar el consejo de curso como una instancia que desarrolla la habilidad de los alumnos para llegar a acuerdos y soluciones. También otro aspecto mencionado por ambos actores educativos es la carencia de una materia específica de formación ciudadana, mencionando que se hace el esfuerzo por incluir estos temas en las diferentes actividades desarrolladas al interior de las escuelas y colegios.

Referente a la forma y los espacios existentes en los diferentes establecimientos para promover lo que se desea de un ciudadano, los docentes indican que la metodología y los momentos en que los desarrolla depende de cada profesor, al no existir una política explícita para esta materia, sin embargo, se reconoce al espacio de consejo de curso como la instancia en donde los alumnos expresan y toman decisiones. También se señala que dentro del liceo/colegio se realizan debates, con el objetivo de fomentar la discusión y el intercambio de opinión. Respecto al tema, los alumnos coinciden con los docentes en considerar al debate y consejo de curso como parte de los espacios que promueven la formación ciudadana; sin embargo, agregan que los docentes con ciertos gestos y demostraciones de preocupación hacia los estudiantes están siendo un ejemplo o modelo a seguir, mencionando además que ellos también pueden entregar este ejemplo a las demás personas, destacando este aspecto como un modo de formación de la ciudadanía al interior de las escuelas. 
En cuanto a las instancias en donde se desarrolla la formación ciudadana, docentes y estudiantes señalan principalmente las votaciones para elegir al centro de alumno, las directivas de curso y la participación en actividades solidarias.

Respecto a los aspectos que obstaculizan la formación ciudada$n a$, los docentes mencionan que la alta cantidad de información, la diversidad y libertad con la que se enfrentan los alumnos los confunde, sin sentirse parte del sistema, o sin saber qué hacer con todos los estímulos que se le presentan. Por otra parte, los profesores no disponen de un tiempo determinado para abarcar temas relacionados con ciudadanía y los estudiantes no tienen interés en estas materias, lo que coincide con lo señalado por los alumnos quienes dicen que no existe la disposición para hablar de estas temáticas. Por último se destaca la opinión de los profesores, quienes indican que este desinterés por parte de los jóvenes se fomenta por el gobierno, a quienes no les convendría promover la discusión y la formación de personas críticas.

Entre las sugerencias entregadas, los docentes mencionan la necesidad de considerar y dialogar sobre ciertas investigaciones empíricas respecto al tema. Por otra parte, se señala la necesidad de interactuar con otras instituciones educativas, que den la oportunidad de conocer diferentes realidades y ejecutar actividades prácticas. Este último punto coincide con lo señalado por los estudiantes quienes plantean la necesidad de salir a la comunidad, ejecutar actividades solidarias, generar espacios al interior de las escuelas, en donde los diferentes cursos compartan y conversen diferentes temas. Por último se indica que se debiera formar criterio político a los alumnos, en donde se traten temas como los diferentes partidos.

En cuanto a los demás espacios en donde se desarrolla la formación de un ciudadano se menciona el hogar. De acuerdo a esto tanto docentes como estudiantes destacan que la base de lo que es una persona parte en la casa con la educación que entregan los padres y se complementa con lo experimentado en la escuela. Por lo tanto, los padres actúan como modelos para sus hijos y, dependiendo del trato y el ejemplo que entregan, determina la forma de ser de cada joven. En lo que respecta a la responsabilidad que tiene el sujeto mismo en su formación como ciudadano se plantea que en general no asume esta tarea, más bien se desvincula o muestra desinterés, sin embargo se destaca que llega un momento en la vida en que cada persona debe hacerse cargo de sus responsabilidades y por sus medios enfrentar desafíos y tomar sus propias decisiones. Finalmente, se menciona el espacio comunitario como un lugar en donde se 
forma la ciudadanía; de acuerdo a esto se indica que las autoridades debieran educar a la sociedad en su conjunto a través de los medios de comunicación y de esta manera adoptar un papel más activo en la formación ciudadana. Por otra parte, los estudiantes señalan que el ciudadano se forma en lo cotidiano y en el compartir con los demás.

\section{CONCLUSIONES Y DISCUSIÓN}

El proceso de construcción de ciudadanía no puede pasar por alto para una sociedad, sea ésta parte de un régimen neoliberal, socialdemocrático o social-libertario. El aparato de la educación formal es, en sí mismo, una instancia donde se promocionan algunas prácticas vinculadas con la ciudadanía y, por ende, un lugar que debe estar en constante cuestionamiento y vigilancia para asegurar que la formación de las futuras generaciones sea a favor de una sociedad más justa, equitativa y libertaria.

El centro de este debate, y lo que hemos podido profundizar gracias a este estudio, es que no se puede escapar de una formación donde los significados transitan desde un enfoque u otro; donde las prácticas ciudadanas y formativas no se encuadran en un tipo solo de ideología, aunque sí es posible vislumbrar acentos más pronunciados según el lugar que se ocupa en la escala de poder de estos sistemas.

El enfoque integrador que presentan Cerda et al. (2004) entre significados liberales y comunitarios, más que una propuesta, emerge como una fisura ideológica (Torres, 1998) propia de los grandes significados sobre la ciudadanía que conviven hoy en nuestra sociedad.

No es que no exista un sentido más acentuado entre ambos enfoques, pero es posible reconocer los significados contaminados de unos con otros, liberales y comunitarios, emancipatorios con neoliberales.

Ya la noción de ciudadanía se construye a partir de relatos de docentes y estudiantes como una mixtura de sueños y expectativas, aunque se observa una ascendencia importante desde el discurso liberal, coherente con el modelo socioeconómico actual. Así, para ambos actores escolares, la ciudadanía se vincularía con una entrega y protección de derechos al individuo, y correspondería con lo que Dueñas (2001) entiende como ciudadanía política e individual, constituida por contenidos formales relacionados con la Educación Cívica, y en donde la «participación» se operacionaliza en el acto de sufragio, que permite la sobrevivencia del modelo democrático imperante. 
Esto no deja de ser llamativo, ya que uno puede entender que el fundamento de esta mirada liberal es buscar mantener el actual modelo, integrar a los individuos al sistema tal cual está, lo que es coherente con el abandono de aquellos elementos ciudadanos relacionados con el debate y el enfrentamiento de ideas (Cerda et al., 2004) que los gobiernos de la Concertación promovieron en educación, incentivando la política de acuerdos y consenso (Portales, 2004; Moulian, 1997). La adaptación o integración del sujeto al modelo social implica hacerlo sin discusión y debate, ya que estos elementos podrían poner en jaque la construcción desde los expertos y técnicos que se instaló en Chile a costa del ejercicio ciudadano (Portales, 2004).

Ahora bien, como ya mencionamos, los significados no son homogéneos en su totalidad, y en ese sentido la subcategoría condiciones para la ciudadanía se conforma con significados más diversos. Aquí, elementos como pertenencia y participación se rescatan por docentes y estudiantes, coincidiendo con enfoques comunitarios (Cerda y cols., 2004) y una ciudadanía Social y Cultural (Dueñas, 2001). Pero la participación se concibe —más por los docentes que por los estudiantes - desde una lógica más formal e institucional, instrumentalizada en el acto del sufragio, como si por el hecho de participar de este rito el/la sujeto se ganasen el derecho a dar su opinión y de cuestionar el propio modelo. Por otro lado, en el bagaje de significados dados por los estudiantes emergen algunos elementos semánticos más flexibles en relación a la participación, pero sin ser el centro del debate.

En esta subcategoría podemos observar uno de los puntos de tensión más interesantes en relación a la oposición adultos/jóvenes, ya que para los primeros además de la mayoría de edad se alude a una integración laboral, para ser considerado un ciudadano pleno; mientras que desde los/as estudiantes aparece una condición contradictoria, ya que reconocen esta condición de mayoría de edad, pero al mismo tiempo se constituyen como ciudadanos por el solo hecho de ser personas. La versión adultocéntrica nos devuelve a una ciudadanía más política formal, individual y por sobre todo neoliberal, este ciudadano consumidor del cual Moulian (1998) hace mención. La versión estudiantil pareciera ser contradictoria, pero en esa fisura se deja entrever, en parte, un sentido de ciudadanía emancipadora, una reivindicación del derecho de transformar la sociedad (Dueñas, 2001) sin límites etarios.

Este tipo de fisuras y contradicciones se hacen más patentes en las subcategorías que valoran la ciudadanía, ya que ambos actores (docentes 
y estudiantes) connotan negativamente este individualismo y las actitudes discriminatorias, propias de un modelo centrado en las libertades individuales (versión extrema del liberalismo); y por su contraparte se connotan positivamente valores relacionados con la solidaridad, dejando ver una mirada positiva — ¿ ¿ nostálgica? - de un acervo comunitarista.

Asimismo, se observa la semejanza semántica con categorías como valores perdidos, que Berumen y Levinson (2007) describieron en sus estudios, con la valoración negativa que realizan los docentes sobre el ciudadano joven, señalándolos como irrespetuosos, desconocedores de sus deberes y de los «valores fundantes» de nuestra sociedad. Este tipo de evaluación se vuelve a reproducir al consultar por los actores escolares en el proceso de formación, dejando entrever nuevamente una mirada esencialmente negativa desde los adultos a los/as estudiantes. Los jóvenes denuncian el maltrato y la exclusión que vivencian en sus establecimientos (especialmente en los municipales).

Desde esta mixtura tensionada de significados de la ciudadanía emergen los significados de la formación ciudadana, el cual puede entenderse que se compone por al menos dos grandes referentes de significados: la influencia de lo que significamos por ciudadanía, y lo que entendemos como proceso formativo.

Así, desde las categorías de sus Objetivos y Contenidos, los docentes y estudiantes aluden a una función de Informar desde la carencia del sujeto formado, o sea, se supone que el sujeto desconoce su rol ciudadano, siendo la escuela el lugar de instrucción.

Pero no es cualquier contenido el centro de preocupación de las comunidades educativas, ya que se centran en elementos sobre los valores y deberes ciudadanos, acercándose a los significados mencionados por Berumen y Levinson (2007) al referirse a los valores perdidos. Además, se plantea esta formación en relación a leyes y normas -operacionalización de los valores mencionados-y de una participación desde las acciones tradicionales de la participación civil (partidos políticos, sufragio, etcétera).

En esencia, se puede reconocer que los significados en juego se conectan especialmente con lo que Cerda et al. (2004) exponen como formación para la ciudadanía política, centrados en la formación sobre la igualdad política y de derechos, para lo cual informar de éstos es fundamental, y donde el acto del sufragio se presenta como el acto ciudadano en esencia. A lo mejor por lo mismo, la coherencia en las prácticas metodológicas que se vinculan con esta formación ciudadana se centra con fuerza en los ritos de las elecciones (Centro de estudian- 
tes, directivas de cursos) y en la exposición frontal de los derechos individuales y sus características.

A pesar de este acento liberal de la ciudadanía actual, se observan fisuras de resistencias a los discursos dominantes, donde se expresan algunos elementos de una formación para la ciudadanía social, más cercana a la línea comunitarista, y que se ejemplifica con que los sentidos a mayor largo plazo terminan aludiendo a un horizonte más solidario y equitativo y una crítica explícita al individualismo.

Por último, la formación ciudadana crítica y activa, aunque emergen con algunos elementos (e.g., metodologías pedagógicas se mencionan debates y análisis, o actividades solidarias), no se presentan como sentidos propios de los establecimientos estudiados en el presente trabajo. En otros términos, no son parte de sus intereses institucionales ya que explícitamente no aparecen en su PEI, ni en su currículo oculto como horizonte velado. Son esencialmente un campo de posibilidades de resistencia que aún no mejora su situación de poder en relación a los demás marcos de significados.

Esto último no es menor, ya que una formación ciudadana centrada en el individualismo, la fragmentación colectiva y la falta de espacios de tomas de decisiones, nos hace vislumbrar una sociedad con similares características, y donde la escuela tiene mucho que decir y aportar en la medida de que esté más consciente de los significados que se transan en lo cotidiano.

VALPARAÍso (CHILE), AGOSTO 2010

RECIBIDO: AGOSTO 2010

ACEPTADO: OCTUBRE 2010

\section{REFERENCIAS BIBLIOGRÁFICAS}

Astudillo, S. y G. CeRDA (2006): «Significado de la formación ciudadana que poseen los docentes de asignaturas pertinentes según el MINEDUC, en establecimientos municipalizados de educación secundaria de la comuna de Valparaíso». Seminario de Título para optar al Título de Psicólogo y Grado de Licenciado en Psicología, Universidad de Valparaíso.

BERUMEN J. y B. LEVINSON (2007): «Educación para una ciudadanía democrática en los países de América Latina: una mirada crítica». REICE. Revista Electrónica Iberoamericana sobre Calidad, Eficacia y Cambio en la Educación, Vol. 5, №4. Disponible en: www.rinace.net. 
Cerda, A.; M. Egaña, A. Magendzo, E. SANTA CRUZ y R. VARAs (2004): El complejo camino de la formación ciudadana: una mirada a las prácticas docentes. Santiago: LOM.

DuEÑAS, C. (2001): «¿Cómo promover la ciudadanía juvenil? Los grupos y asociaciones juveniles como un espacio de educación ciudadana». Disponible en: www.interjoven.cl.

GARRETón, M. (2000): Política y jóvenes en Chile. Santiago: Fundación Ebert.

GIROUX, H. (1992): «La escuela y la política del currículum oculto». En Teoría y resistencia en educación. México: Siglo XXI.

_ (1983): «Teorías de la reproducción y la resistencia en la nueva sociología de la educación: un análisis crítico». Revista Dialogando, Nº10.

ICCS (2010): «Estudio internacional de educación cívica y formación ciudadana: primer informe nacional de resultados». Disponible en: www.simce.cl.

InJuv (2010): Sexta encuesta nacional de juventud. Principales resultados. Disponible en: www.injuv.cl.

- (2006): Segundo informe nacional de juventud. Disponible en: www.injuv.cl.

MARTínEZ, M. (1998): El contrato moral del profesorado: condiciones para una nueva escuela. Madrid: Editorial Desclée de Brouwer.

MCMillan, J. y S. SCHumaCHER (2005): Investigación educativa. Madrid: Editorial Pearson Educación.

MeLLA, O. (2000): «Grupos focales: técnica de investigación cualitativa». Serie Documentos CIDE. Santiago: CIDE.

MiNEDUC (2004a): «Informe de la comisión sobre formación ciudadana». Disponible en: www.mineduc.cl.

- (2004b): «Formación ciudadana en el currículum de la reforma». Disponible en: www.mineduc.cl.

Moulian, T. (1999): El consumo me consume. Santiago: LOM.

- (1997): Chile actual: anatomía de un mito. Santiago: LOM.

PORTALES, F. (2000): Chile: una democracia tutelada. Santiago: Sudamericana.

SALAMANCA, A. y C. MARTíN-CRESPO (2007): «El diseño en la investigación cualitativa». Departamento de Investigación de la Fundación para el Desarrollo de la Enfermería. Disponible en: www.nureinvestigacion.es

SERVICIO ELECTORAL (2006): «Estadísticas de padrón: estadísticas comunales». Disponible en: www.servel.cl.

STRAUSS, A. Y J. CORBIN (2002): Bases de la investigación cualitativa: técnicas y procedimientos para desarrollar la teoría fundamentada. Medellín: Editorial Universidad de Antioquia.

THEZÀ, M. (2003): «Apuntes para una resignificación de la participación política de los jóvenes a partir del eje igualdad/desigualdad». Última Década $\mathrm{N}^{\circ} 19$. Viña del Mar: Ediciones CIDPA.

TORRES, J. (1998): El currículum oculto. Madrid: Editorial Morata. 